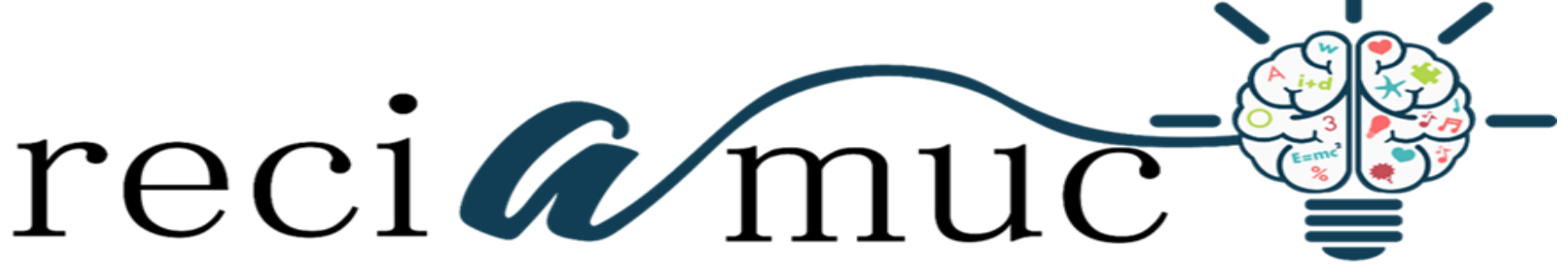

Revista cientifica de investigación actualización del mundo de las ciencias

Mónica Monserrate Solórzano Vélez a ; Iván Andrés Jaramillo Chávez ${ }^{\text {b; Flor }}$

María Alcívar Chávez c ; Daniela Stefanía Cedeño Ching d; Iris Galicia Cevallos

Macías $^{\text {e; }}$ María Belén Iglesias López ${ }^{\text {f }}$

Trastornos causados por el consumo de sustancias derivadas del cannabis

Disorders caused by the use of substances derived from cannabis

Revista Científica de Investigación actualización del mundo de las Ciencias. Vol.

3 núm., 3, julio, ISSN: 2588-0748, 2018, pp. 1486-1502

DOI: $\underline{\text { 10.26820/reciamuc/3.(3).julio.2019.1486-1502 }}$

URL: http://reciamuc.com/index.php/RECIAMUC/article/view/406

Código UNESCO: 3205 Medicina Interna

Tipo de Investigación: Artículo de Investigación

(C) RECIAMUC; Editorial Saberes del Conocimiento, 2019

Recibido: 28/04/2019

Aceptado: 19/05/2019

Publicado: 01/07/2019

Correspondencia: monikiss 1990@ hotmail.com

a. Médica Cirujana; Centro de salud Chone tipo C Distrito 13D07 Chone - Flavio Alfaro - Ecuador; monikiss_1990@hotmail.com

b. Médico Cirujano; Hospital de especialidades Portoviejo; Portoviejo - Ecuador; jaramilloivan_08@hotmail.com

c. Médica Cirujana; Centro de salud Chone tipo C Distrito 13D07 Chone - Flavio Alfaro - Ecuador; floralcivar92@outlook.com

d. Médica Cirujana; Médico a domicilio ayuda salud y RESCARE en Santiago Región metropolitana, Médico en Clínica Digital Santiago Chile - Chile; danielaceching_90@ hotmail.es

e. Médica Cirujana; Centro de Salud Chone tipo C Distrito 13D07 Chone - Flavio Alfaro - Ecuador; galycevallos16@gmail.com

f. Médica Cirujana; IESS Campesino Dispensario Pasadero Distrito 13D14; Portoviejo - Ecuador; belen_iglesias92@hotmail.com 


\section{Trastornos causados por el consumo de sustancias derivadas del cannabis}

Vol. 3, núm. 3., (2019)

Mónica Monserrate Solórzano Vélez; Iván Andrés Jaramillo Chávez; Flor María Alcívar Chávez; Daniela Stefanía Cedeño Ching; Iris Galicia Cevallos Macías; María Belén Iglesias

\section{RESUMEN}

El presente trabajo de investigación abarca el estudio de los trastornos causados por el cannabis y las psicoterapias que se pueden emplear para ser tratadas a las personas que han consumido este psicotrópico. El objetivo es documentar los trastornos por consumo de derivados del cannabis dentro de la salud mental. Se emplea la metodología descriptiva transversal a través de una revisión sistemática, se utilizaron técnicas de selección mediante criterios de inclusión y exclusión. Para los criterios de inclusión se optó por artículos destinados al estudio del trastorno en la salud mental que resultan por el consumo de cannabis y de sus sustancias derivadas, a través, de la identificación de datos de revistas. La idea es dar a conocer si existe una relación directa entre el consumo de cannabis y la aparición de trastornos psicológicos en el organismo. El trabajo se divide en tres fragmentos la parte inicial abarcara información general del cannabis, denominación y principal principio activo de dependencia; en la segunda parte se describen los tipos de trastorno que provoca el consumo de esta sustancia bien sean por consumo o inducidos; y la tercera parte incluye la discusión y conclusión del trabajo investigativo.

Palabras Claves: Cannabis; Trastornos; Consumo; Sustancias. 


\section{Trastornos causados por el consumo de sustancias derivadas del cannabis}

Vol. 3, núm. 3., (2019)

Mónica Monserrate Solórzano Vélez; Iván Andrés Jaramillo Chávez; Flor María Alcívar

Chávez; Daniela Stefanía Cedeño Ching; Iris Galicia Cevallos Macías; María Belén Iglesias

López

\section{ABSTRACT}

This research paper covers the study of cannabis disorders and psychotherapies that can be used to treat people who have used this psychotropic. The objective is to document the disorders caused by cannabis derivatives in mental health. The transversal descriptive methodology is used through a systematic review, selection techniques were used using inclusion and exclusion criteria. For the inclusion criteria, articles were chosen for the study of mental health disorder that result from the use of cannabis and its substances derived, through the identification of journal data. The idea is to make known if there is a direct relationship between cannabis use and the appearance of psychological disorders in the body. The work is divided into three fragments, the initial part will cover general cannabis information, name and main active principle of dependence; the second part describes the types of disorder that causes the consumption of this substance, whether by consumption or induced; and the third part includes the discussion and conclusion of the research work.

Key Words: Cannabis; Disorders; Consumption; Substances. 


\section{Trastornos causados por el consumo de sustancias derivadas del cannabis}

Vol. 3, núm. 3., (2019)

Mónica Monserrate Solórzano Vélez; Iván Andrés Jaramillo Chávez; Flor María Alcívar Chávez; Daniela Stefanía Cedeño Ching; Iris Galicia Cevallos Macías; María Belén Iglesias

López

\section{Introducción.}

El cannabis tiene diferentes denominaciones Cannabis sativa, Cannabis indica y Cannabis ruderalis de acuerdo a diferentes fuentes se establece que es la misma planta, solo varían en ciertas características en cuanto al tamaño y a la apariencia debido al clima y al suelo. Alrededor del mundo se la conoce con diferentes nombres entre al más popular la marihuana u otros nombres propios de acuerdo a la cultura local. (Organization, 2018)

Además, el cannabis tiene diferentes formas de consumo y maneras de preparación. A nivel mundial el consumo de cannabis es alarmante en jóvenes, ya sea por la adicción o por curiosidad, se ha demostrado, que el consumo disminuye por ciclos de vida como la adultez o el envejecimiento debido a las responsabilidades que optan como el matrimonio, empleo o la vejez.

El cannabis o marihuana se define como una droga que produce dependencia y adicción, actualmente se conoce que su dependencia es un proceso psicológico que se traduce en cambios neuronales en el cerebro; dependencia psíquica y dependencia física son las dos caras de una misma moneda. Al fumarse, alcanza en pocos segundos el cerebro un factor farmacocinético que facilita el desarrollo de la dependencia. (E, Casavielles, Herse, \& J, 2017)

El consumo prolongado del cannabis altera los circuitos cerebrales debido a su principal componente psicoactivo que es el delta-9-tetrahidrocannabinol (THC). Sin embargo, el cannabis contiene más de 70 cannabidivarin, la tetrahidrocannabivarin y los terpenoides. Muchos de estos compuestos tienen efecto farmacológico distintos de los del THC. (T, WilkinsonRajiv, RadhakrishnanDeepak, \& D’Souza, 2016)

El tethahidrocanabinol, se fija a la estructura química específica de la membrana neuronal. En el hombre, la marihuana se desliga lentamente de los receptores, por lo que los síntomas de privación, como irritabilidad, y agresividad son menos evidentes, pero si, suficiente efectivo para que la persona persista en el consumo. (E, Casavielles, Herse, \& J, 2017) 


\section{Trastornos causados por el consumo de sustancias derivadas del cannabis}

Vol. 3, núm. 3., (2019)

Mónica Monserrate Solórzano Vélez; Iván Andrés Jaramillo Chávez; Flor María Alcívar

Chávez; Daniela Stefanía Cedeño Ching; Iris Galicia Cevallos Macías; María Belén Iglesias López

En la actualidad hay evidencias de que esta droga provoca por largo tiempo un aumento de la frecuencia cardiaca y la presión arterial, además produce relajamiento bronquial, tos crónica, bronquitis, cáncer pulmonar, conjuntivitis, disminución del número movilidad de los espermatozoides (infertilidad), disfunción eréctil, anomalías cromosómicas, menor concentración de testosterona, así con inhibición de la síntesis del ácido ribonucleico (RNA) y el ácido desoxirribonucleico (ADN), entre otras. (Med, 2018)

Dentro de los efectos adversos por el consumo crónico en el área psicosocial se encuentra, que el uso regular de la droga puede inducir un síndrome de dependencia en uno de cada diez consumidores, y en uno de cada seis, si su inicio fue en la adolescencia. Sin embargo, el uso regular de cannabis duplica el riesgo de experimentar síntomas psicóticos, especialmente si existe presencia de una historia de trastornos psicóticos y si inician su consumo antes de los 15 años. (Med, 2018)

La mejor evidencia de los efectos psicotomiméticos agudos del cannabis proviene de estudios experimentales con cannabis sintéticos, que producen una gama completa de síntomas positivos (como desconfianza, ilusiones paranoicas, desorganización conceptual, pensamientos fragmentados y alteraciones perceptivas), síntomas negativos (como afecto embotamiento, abstinencia emocional, retraso psicomotor, falta de espontaneidad y relación reducida, y trastornos cognitivos (como deficiencias en el aprendizaje verbal, memoria corto plazo, memoria de trabajo, función ejecutiva, capacidad abstracta, toma de decisiones, atención y anomalías en la percepción del tiempo) en voluntarios sanos que se desaparecen a los síntomas de la esquizofrenia crónica. Además, el THC exacerba los síntomas psicóticos en pacientes con esquizofrenia crónica, a pesar de estar en dosis estables de antipsicóticos. (T, WilkinsonRajiv, RadhakrishnanDeepak, \& D'Souza, 2016)

El consumo de cannabis es un fenómeno ampliamente extendido en nuestra sociedad. Aunque no están suficientemente dilucidadas las alteraciones psicopatológicas inducidas por el consumo de cannabis, con los conocimientos actuales se puede afirmar que el consumo de cannabis no es inocuo para la salud. (Casas, 2017) 


\section{Trastornos causados por el consumo de sustancias derivadas del cannabis}

Vol. 3, núm. 3., (2019)

Mónica Monserrate Solórzano Vélez; Iván Andrés Jaramillo Chávez; Flor María Alcívar Chávez; Daniela Stefanía Cedeño Ching; Iris Galicia Cevallos Macías; María Belén Iglesias

Los trastornos por consumo de cannabis son un espectro de afecciones clínicamente importantes, y se define conforme a criterios psicológicos, sociales y fisiológicos para documentar las consecuencias adversas, la pérdida de control del consumo y los síntomas de abstinencia. Los trastornos por consumo de cannabis se definen en el Manual Diagnóstico y Estadístico de los Trastornos Mentales y en la Clasificación Estadística Internacional de Enfermedades y Problemas Relacionados con la salud. La CIE- 10 distingue entre el consumo nocivo de cannabis y el consumo de cannabis con dependencia, mientras que en el DSM-V los trastornos por consumo de cannabis se clasifican, según la gravedad de la afectación, también describen un síndrome especifico de abstinencia del cannabis que puede tener lugar en un plazo de 24 horas desde el consumo. Para diagnosticar un síndrome de abstinencia de cannabis, la persona afectada debe referir al menos dos síntomas mentales (por ejemplo, irritabilidad, inquietud, ansiedad, depresión, agresividad, pérdida de apetito, trastornos del sueño) y al menos un síntoma físico (por ejemplo, dolor, temblores, sudación, temperatura corporal elevada, escalofríos). Estos síntomas son más intensos en la primera semana de abstinencia, pero pueden persistir hasta un mes. (Organization, 2018)

El manual diagnosticado y estadístico de los trastornos mentales clasifican a los trastornos producidos por cannabis por consumo e inducidos, esta clasificación excluye al síndrome de abstinencia a pesar que este tipo de psicopatología presenta gran relevancia clínica dentro del grupo de trastornos ya escritos.

Dentro de los trastornos por consumo de cannabis se encuentra la dependencia y abuso; y dentro de los trastornos inducidos por cannabis: intoxicación, alteraciones perceptivas, el delirium por intoxicación por cannabis, el trastorno psicótico inducido por cannabis con ideas delirantes, el trastorno psicótico inducido por cannabis con alucinaciones y el trastorno de ansiedad inducido por cannabis. (Royo \& 1981, 2014)

\section{Trastornos por consumo de cannabis}

- Dependencia de cannabis: Los sujetos con dependencia de cannabis presentan un consumo compulsivo y en general no desarrollan dependencia fisiológica, aunque la 


\section{Trastornos causados por el consumo de sustancias derivadas del cannabis}

Vol. 3, núm. 3., (2019)

Mónica Monserrate Solórzano Vélez; Iván Andrés Jaramillo Chávez; Flor María Alcívar

Chávez; Daniela Stefanía Cedeño Ching; Iris Galicia Cevallos Macías; María Belén Iglesias

López

tolerancia a muchos de los efectos de cannabis ha sido observada en sus consumidores crónicos. También se han observado algunos síntomas de abstinencia, pero sin significación clínica. Los sujetos con dependencia de cannabis consumen abundantes cantidades a lo largo del día durante meses o años e invierten muchas horas para adquirir y consumir la sustancia, lo que interfiere con frecuencia las actividades sociales, laborales o recreativas, los sujetos con dependencia de cannabis pueden persistir en el consumo a pesar de conocer los problemas físicos ( por ejemplo, tos crónica relaciona con fumar tabaco) o los problemas psicológicos (por ejemplos, sedación excesiva como resultado del consumo de dosis altas) por ello comporta. (Pichot, Aliño, \& Miyar, 2017)

La definición de la dependencia de cannabis es muy similar en las clasificaciones imperantes. Clásicamente al conceptuar la dependencia de sustancias se ha primado la existencia de un síndrome de abstinencia y tolerancia. Siendo criterios muy importantes se debe destacar que la no existencia de algunos de ellos, o de los dos, no excluye necesariamente la existencia de un cuadro de dependencia. (Artamendi, R, Hermida, Secades-Villa, \& Garcia-Portilla, 2016)

- Abuso de cannabis. El consumo periódico y la intoxicación por cannabis pueden interferir las actividades laborales o escolares y ser peligroso en situaciones como la conducción de vehículos. Puede haber problemas legales como consecuencias de detenciones por posesión de cannabis. Hay discusiones con la esposa u otros familiares acerca de la posesión de cannabis en casa o de su consumo en presencia de niños. Cuando hay niveles significativos de tolerancia, o cuando los problemas físicos se asocian con el consumo compulsivo de cannabis, debe considerarse el diagnóstico de dependencia en lugar del abuso. (López-Ibor, 2015)

\section{Trastornos inducidos por cannabis}

- Intoxicación por cannabis. Se denomina intoxicación los cambios conductuales o comportamentales, clínicamente significativos que se presentan tras el consumo de cannabis. La intoxicación se inicia con una sanción de bienestar, siendo máxima a los 1030 minutos, cuando los niveles plasmáticos son mayores. La característica esencial de la 


\section{Trastornos causados por el consumo de sustancias derivadas del cannabis}

Vol. 3, núm. 3., (2019)

Mónica Monserrate Solórzano Vélez; Iván Andrés Jaramillo Chávez; Flor María Alcívar Chávez; Daniela Stefanía Cedeño Ching; Iris Galicia Cevallos Macías; María Belén Iglesias

López

intoxicación por cannabis es la presencia de cambios psicológicos o comportamentales clínicamente significativos que aparecen durante o poco tiempo después del consumo de cannabis. La intoxicación inicia típicamente con una sensación de bienestar (high) seguida de síntomas que incluyen euforia con risas inapropiadas y grandiosidad, sedación, letargia, deterioro de la memoria inmediata, dificultades para llevar a cabo procesos mentales complejos, deterioro de la capacidad de juicio, percepciones sensoriales distorsionadas, deterioro de la actividad motora y sensación de que el tiempo trascurre lentamente. Ocasionalmente, aparece ansiedad (que puede ser grave), disforia o retraimiento social. Estos efectos psicoactivos se acompañan de dos o más de los siguientes signos, que se presentan a las 2 horas del consumo de cannabis: irritación conjuntival, aumento del apetito, sequedad de boca y taquicardia. Los síntomas no son debidos a enfermedad medica no se explican mejor por la presencia de otro trastornomental. (Loirth, 2017)

- Intoxicación por cannabis, con alteraciones perceptivas. Este diagnóstico puede realizarse cuando los criterios coinciden con intoxicación por cannabis y alucinaciones auditivas, visuales o táctiles, con juicio de realidad intacto, en ausencia de un delirium. Juicio de realidad intacto significa que el sujeto sabe que las alucinaciones son inducidas por la sustancia y no representan la realidad externa. Cuando las alucinaciones aparecen en ausencia de juicio de realidad intacto debe considerarse el diagnóstico de trastorno psicótico inducido por sustancias. (Dimith, 2016)

- Delirium por intoxicación por cannabis. El cannabis puede producir delirium tras el consumo de grandes cantidades. Es raro y se suele producir en el contexto de policonsumo, consumo masivo o con consumidores nóveles. Cursa con un cuadro confusional, con alteraciones amnésicas y sintomatología de ansiedad, es indistinguible de los cuadros confunsionales de otras etiologías y su curso suele ser auto limitado. (Casas, 2017)

El delirium que aparece durante la intoxicación por sustancias puede desarrollarse minutos u horas después de haber ingerido dosis relativamente altas de algunas drogas como cannabis, cocaína y alucinógenos. Con otras sustancias como el alcohol, los 


\section{Trastornos causados por el consumo de sustancias derivadas del cannabis}

Vol. 3, núm. 3., (2019)

Mónica Monserrate Solórzano Vélez; Iván Andrés Jaramillo Chávez; Flor María Alcívar

Chávez; Daniela Stefanía Cedeño Ching; Iris Galicia Cevallos Macías; María Belén Iglesias López

barbitúricos o las meperidia, el delirium a veces solo se presenta después de una intoxicación mantenida durante algunos días. Con frecuencia, el delirium se resuelve en pocas horas o días cuando la intoxicación cede (aunque la duración puede prolongarse tras la intoxicación con fenciclidina.) (Gainza, Nogué, Velasco, Hoffman, Burillo-Putze, \& Dueñas., 2015)

Trastorno psicótico inducido por cannabis, con ideas delirantes.

Las características esenciales del trastorno psicótico inducido por sustancias en la presencia de alucinaciones o ideas delirantes (criterio A) que se considera como efectos fisiológicos directos de algunas sustancias (droga, medicamento o exposición a un toxico). No se incluyen las alucinaciones cuando el sujeto es consciente de que son provocadas por una sustancia o abstinencia de sustancia, con la especificación de si hay o no alteraciones perceptivas. (Pichot, Aliño, \& Miyar, 2017)

La alteración no se explica mejor por la presencia de un trastorno psicótico no inducido por sustancias. No se establece el diagnostico debe realizarse en lugar del de intoxicación por sustancia o abstinencia solo si los síntomas son excesivos en relación con los habitualmente asociado con el síndrome de intoxicación o de abstinencia, y cuando los síntomas son de suficiente gravedad como para merecer atención clínica independiente. El trastorno psicótico inducido por sustancias se distingue de un trastorno psicótico primario teniendo en cuenta el inicio, el curso y otros factores. (Pichot, Aliño, \& Miyar, 2017). En cuanto a las drogas de abuso, tiene que haber pruebas de intoxicación o abstinencia en la historia clínica, la exploración física o lo hallazgos de laboratorio. Los trastornos psicóticos inducidos por sustancias solo se producen en asociación con estados de intoxicación o abstinencia, mientras que los trastornos psicóticos primarios pueden preceder el inicio del consumo de la sustancia o puede producirse tras largos periodo de abstinencia. Una vez iniciados, los síntomas psicóticos pueden continuar mientras continúe el consumo de la sustancia. Debido a que en algunas sustancias el síndrome de abstinencia puede aparecer con relativo retraso, el inicio de los síntomas psicóticos puede darse hasta 4 semanas después del abandono de la sustancia. Otra consideración es la referente a la presencia de características atípicas para un trastorno psicótico primario (p. ej.., edad de inicio o 


\section{Trastornos causados por el consumo de sustancias derivadas del cannabis}

Vol. 3, núm. 3., (2019)

Mónica Monserrate Solórzano Vélez; Iván Andrés Jaramillo Chávez; Flor María Alcívar Chávez; Daniela Stefanía Cedeño Ching; Iris Galicia Cevallos Macías; María Belén Iglesias

curso atípicos). Por ejemplo, la aparición por primera vez de ideas delirante en una persona mayor de 35 años, sin una historia conocida de un trastorno psicótico primario, debe alentar al clínico sobre la posibilidad de que se encuentre ante un trastorno psicótico inducido por sustancias. (Pichot, Aliño, \& Miyar, 2017)

Incluso la historia previa de un trastorno psicótico primario no descarta la posibilidad de un trastorno psicótico inducido por sustancias. Se ha sugerido que 9 de cada 20 alucinaciones no auditivas son productos de un trastorno psicótico inducido por sustancias o de un trastorno psicótico debido a la enfermedad médica. (Pichot, Aliño, \& Miyar, 2017) Por el contrario, los factores que sugieren que los síntomas psicóticos son atribuibles a un trastorno psicóticos primario incluyen la persistencia de los síntomas psicóticos durante un periodo sustancial de tiempo (p. ej.., alrededor de 2 mes) tras el final de la intoxicación o la abstinencia de la sustancia, la aparición de síntomas que son claramente excesivos en relación con lo que cabría esperar por el tipo o la cantidad de la sustancia utilizada o la duración del uso, o una historia de trastornos psicóticos primarios recidivantes. Incluso en una persona con intoxicación o abstinencia de han de tener en cuenta otras posibles causas de síntomas psicóticos, a que los problemas por consumo de sustancia no son raros entre las personas con trastornos psicóticos (presumiblemente) no inducidos por sustancias (Pichot, Aliño, \& Miyar, 2017)

\section{Subtipos y especificaciones}

Pueden emplearse los siguientes subtipos para indicar el síntoma predominante en el cuadro. Si hay ideas delirantes y alucinaciones, se debe codificar lo que predomine: (Pichot, Aliño, \& Miyar, 2017)

- Con ideas delirantes. Este subtipo se emplea si predominan las ideasdelirantes.

- Con alucinaciones. Este subtipo se emplea sin predominan las alucinaciones. (Pichot, Aliño, \& Miyar, 2017)

El contexto en el que se hayan iniciado los síntomas psicóticos pueden indicarse utilizando las especificaciones siguientes: 


\section{Trastornos causados por el consumo de sustancias derivadas del cannabis}

Vol. 3, núm. 3., (2019)

Mónica Monserrate Solórzano Vélez; Iván Andrés Jaramillo Chávez; Flor María Alcívar

Chávez; Daniela Stefanía Cedeño Ching; Iris Galicia Cevallos Macías; María Belén Iglesias

López

- De inicio durante la intoxicación. Se debe usar esta especificación si se cumplen los criterios para la intoxicación por las sustancias y los síntomas aparecen durante el síndrome durante el síndrome de intoxicación. (Pichot, Aliño, \& Miyar, 2017)

- De inicio durante la abstinencia. Se debe usar esta especificación si se cumplen los criterios para la abstinencia de la sustancia y si los síntomas aparecen durante o poco después del síndrome de abstinencia. (Pichot, Aliño, \& Miyar, 2017)

Trastorno de ansiedad inducido por cannabis. El consumo de cannabis está relacionado con la aparición de síntomas de ansiedad que en algunos pacientes pueden llegar a constituir auténticas crisis de ansiedad (Casas, 2017). Su aparición está relacionada con consumidores noveles o con la intensidad del consumo. Los trastornos de ansiedad y efectivos son los más frecuentes en consumidores de cannabis. La sobredosis de cannabis no es mortal, cuando el consumo es exclusivo de estas sustancias. Cursa con taquicardia, ataxia, temblor, labilidad efectiva, y aumento súbito de la ansiedad. En ocasiones la sintomatología puede llegar a ser similar a un ataque de pánico. (Lorente, Actis, López-Cánovas, \& Hernández-Torrado, 2016)

En la evaluación las pruebas de laboratorio de orina, sangre, saliva o cabello pueden ser útiles para detectar el uso de cannabis. Los ensayos generalmente se basan en la detección del metabolito activo más común, el ácido delta 9-tetraahidrocannibinolitico. Se ha estudiado a fondo, y este metabolito acido abundante se ha convertido en un mercado urinario establecido del consumo de cannabis en análisis forenses, clínicos y ambientales. Los fumadores de cannabis pesados o crónicos tardaran más en eliminar el THC en comparación con los usuarios esporádicos o de una sola vez, Además, otras pruebas para descartar condiciones adicionales pueden se beneficios. Esto incluye imágenes de la cabeza, o pruebas de laboratorio de metales pesados, infección y marcadores inmunológicos, alteraciones de electrolitos u hormonas. (Patel \& Marwaha., 2019)

El objetivo del tratamiento se enfoca en el manejo de la función general del individuo que es multifasica y multifactorial. Puede proporcionarse tratamiento de apoyo durante la desintoxicación; permite el acceso a servicio psiquiátricos permite abordar trastornos subyacentes; el asesoramiento psicológico puede modificar el comportamiento, desarrollar 


\section{Trastornos causados por el consumo de sustancias derivadas del cannabis}

Vol. 3, núm. 3., (2019)

Mónica Monserrate Solórzano Vélez; Iván Andrés Jaramillo Chávez; Flor María Alcívar Chávez; Daniela Stefanía Cedeño Ching; Iris Galicia Cevallos Macías; María Belén Iglesias

López

habilidades de afrontamiento más saludables frente a los factores de estrés e iluminarlos respecto a su temperamento. (Patel \& Marwaha., 2019)

A medida que las variedades de cannabis se vuelven más potentes y accesibles, el riesgo aumentara por la frecuencia y la gravedad de las reacciones adversas graves. Para las personas con marcada intoxicación o abstinencia, o trastorno por consumo de cannabis, el objetivo debe ser el cese de la droga por completo. Una disminución gradual en lugar de un cese abrupto es probable que disminuya la incomodidad de la abstinencia y prevenga la recaída. La intoxicación por cannabis con mayor frecuencia no requiere tratamiento médico y se resolverá por sí misma. (Patel \& Marwaha., 2019)

El manejo de apoyo, como un ambiente tranquilo y no estimulante, ayuda a los pacientes. El tratamiento sintomático se puede considerar para los síntomas específicos, como los agonista alfa-2-2adrenergicos o los betabloqueantes para la taquicardia, los benzodiacepinas para los ataques de pánico. El uso no autorizado de antihistamínicos de primera generación para la ansiedad y la inquietud y los neurolépticos para la psicosis. La desintoxicación farmacológica todavía estaba bajo investigación. Una revisión sistémica indica que la mayoría de los estudios son preliminares y no pueden respaldar estadísticamente las razones clínicas, ya que son de tamaño, inconsciente y tiene un riesgo de sesgo de desgaste. (Patel \& Marwaha., 2019)

\section{Metodología.}

Para la elaboración de la presente investigación se realizó una revisión sistemática de artículos de revistas científicas, aplicando el método descriptivo transversal mediante la técnica de criterios de inclusión y de exclusión, se procedió a la selección de documentos de revistas publicados en los últimos cincos años.

De manera más explicativa se diseñó y ejecuto una revisión sistémica con el objetivo de identificar los trastornos causados por el consumo de cannabis, se realizó una búsqueda en la base de datos de las revistas de Scielo, Elseiver, revistas médica, Medline y shilder, utilizando 


\section{Trastornos causados por el consumo de sustancias derivadas del cannabis}

Vol. 3, núm. 3., (2019)

Mónica Monserrate Solórzano Vélez; Iván Andrés Jaramillo Chávez; Flor María Alcívar

Chávez; Daniela Stefanía Cedeño Ching; Iris Galicia Cevallos Macías; María Belén Iglesias

López

términos de trastornos, alteración de la salud mental, efectos sobre la salud mental, sin restricciones de la población, edad, sexo, ocupación, etc.

Se encontraron 15 artículos de los cuales solo cumplían con los criterios de inclusión 5 artículos, los mismos que permitieron verificar los trastornos más comunes por el consumo de la sustancia además nos permitió aclarar ideas sobre la dependencia y la adicción.

\section{Discusión}

De acuerdo a la revisión bibliográfica se procedió a la elección de las variables de estudio extraído de los documentos previamente revisados. Se puede establecer que el cannabis es la sustancia más conocida a nivel mundial haciendo énfasis en los jóvenes por mayor grado de consumo y dependencia, el mismo que disminuye de acurdo al ciclo de vida y rol que toma de acuerdo a las etapas del desarrollo personal.

Acorde al uso en edades tempranas encontramos una revisión de estudios que concluye que las alteraciones cognitivas por cannabis se pueden producir tras un uso muy prolongado, quizá superior a los 15 años. (Tziraki, 2017)

A diferencia de otro artículo en donde recientemente, varios estudios importantes han contribuido a la evidencia de una asociación entre el consumo de cannabis y el deterioro cognitivo persistente. Un estudio notable fue un seguimiento de la cohorte de Dunedin, donde se evaluó el consumo de cannabis mediante entrevistas y se realizaron pruebas neuropsicológicas a los 13 y 38 años de edad. Los usuarios de cannabis mostraron una disminución neurocognitiva en numerosos dominios, incluida la velocidad de procesamiento, la memoria, el funcionamiento ejecutivo y la comprensión verbal. (T, WilkinsonRajiv, RadhakrishnanDeepak, \& D’Souza, 2016)

Se puede determinar que en ambos estudios después de dejar de consumir la sustancia los efectos no fueron reversibles, pero si se han logrado controlarlos gracias a diferentes terapias. 


\section{Trastornos causados por el consumo de sustancias derivadas del cannabis}

Vol. 3, núm. 3., (2019)

Mónica Monserrate Solórzano Vélez; Iván Andrés Jaramillo Chávez; Flor María Alcívar Chávez; Daniela Stefanía Cedeño Ching; Iris Galicia Cevallos Macías; María Belén Iglesias

López

En otro estudio reciente, Fontes investigo el rendimiento neurocognitivo de los consumidores crónicos de cannabis y los controles sanos. Los usuarios de cannabis que comenzaron a usar antes de los 15 años tuvieron un desempeño peor que los controles en las medidas de atención sostenida, control de impulso y funcionamiento ejecutivo. (T, WilkinsonRajiv, RadhakrishnanDeepak, \& D’Souza, 2016)

Se han estudiado varios genes candidatos que interactúan con la exposición al cannabis para conferir un mayor riesgo de esquizofrenia. La catecol-O-metiltransferasa (COMT) es una enzima que metaboliza la dopamina en la corteza prefrontal; los individuos con el genotipo Val/Val tienen una mayor actividad metabólica de esta enzima (y, por lo tanto, un nivel más bajo de dopamina cortical prefrontal) en relación con aquellos con el polimorfismo Met/ Met. (T, WilkinsonRajiv, RadhakrishnanDeepak, \& D'Souza, 2016)

Los primeros informes sugirieron que los individuos con el genotipo Val/ Val y COMT tenían diez veces más probabilidades de desarrollar psicosis que aquellos con el genotipo Met/Met sin embargo, los datos posteriores han arrojado resultados mixtos. (T, WilkinsonRajiv, RadhakrishnanDeepak, \& D’Souza, 2016)

De acuerdo a estudios de interacción genético de cannabis Caspi en el 2005 en un muestra de 03 de 26 años detalla que desarrolla trastornos psicóticos, Zaamit en 2011 en una muestra de 2060 encontró psicosis en los consumidores de cannabis; mientras que costas en 2011 en un estudio de caso único mediante un análisis transversal con una muestra 748 de acuerdo a la historia del consumo del cannabis encontró pacientes con esquizofrenia en donde se atribuyó que esta sustancia era un desencadenante de esta patología pero aún faltan estudios para confirmarla. (T, WilkinsonRajiv, RadhakrishnanDeepak, \& D’Souza, 2016)

Existen varios estudios que demuestran que el consumo prolongado afecta a la salud mental, en los estudios referidos anteriormente se vincula al consumo de cannabis diferentes trastornos entres los más relevantes el trastorno de dependencia, la psicosis y la esquizofrenia en esta última faltan aún estudio por realizar. 


\section{Trastornos causados por el consumo de sustancias derivadas del cannabis}

Vol. 3, núm. 3., (2019)

Mónica Monserrate Solórzano Vélez; Iván Andrés Jaramillo Chávez; Flor María Alcívar

Chávez; Daniela Stefanía Cedeño Ching; Iris Galicia Cevallos Macías; María Belén Iglesias

López

\section{Conclusión.}

El cannabis contiene una variedad de sustancias, su principal principio activo es el delta 9 tetrahidrocannabimol (THC) capaz de pasar la barrera hematocefalica y hemato placentaria siendo el más adictivo, provocando alteraciones a nivel cognoscitivo, psicomotor y conductual, el consumo de estas sustancias a altas dosis conlleva a la alteración a nivel mental produciendo trastornos de dependencia, psicosis; adicción y dependencia es decir trastornos por consumo o trastorno inducidos por el consumo de cannabis.

Después de una sola instancia de intoxicación, los efectos psicotóxicos agudos causados por fumar cannabis en las funciones cognitivas (capacidad de razonamiento, funciones de memoria, capacidad de análisis y planificación, etc.) permanecerán, en general, por un máximo de cuatro a cinco horas. La duración de estos efectos depende del nivel de THC en la sangre, y hay un retraso de aproximadamente una hora en relación con el tiempo de consumo. En el caso de consumo repetido, es decir, en una o más ocasiones por día, los impedimentos funcionales persistirán (aunque el individuo aprenda cómo ocultar ciertas deficiencias funcionales) y toda la personalidad eventualmente quedará marcada por sobre todo cognitivas dificultades, y también por las estrategias sociales a las que el individuo debe recurrir para hacer frente.

Durante el desarrollo de la investigación se observó que la disminución de la capacidad para llevar a cabo complejos operaciones de pensamiento, capacidad reducida para concentrarse, capacidad disminuida para procesar información, deterioro de la memoria a corto plazo, reducción, flexibilidad intelectual y capacidad de aprender de la experiencia, bajada de la capacidad de llevar a cabo una planificación estratégica a largo plazo y dificultades para expresarse verbalmente en situaciones nuevas y desconocidas en las que los viejos modos de pensamiento y los viejos conocimientos son inadecuados, son parte de las manifestaciones de lo crónico y los efectos de este psicotrópico.

El abuso crónico también conduce a un deterioro medible en el "sentido de coherencia" de los individuos (es decir, su percepción y comprensión de su entorno), aunque este efecto se 


\section{Trastornos causados por el consumo de sustancias derivadas del cannabis}

Vol. 3, núm. 3., (2019)

Mónica Monserrate Solórzano Vélez; Iván Andrés Jaramillo Chávez; Flor María Alcívar Chávez; Daniela Stefanía Cedeño Ching; Iris Galicia Cevallos Macías; María Belén Iglesias

desvanece semanas después de que se interrumpa el abuso, o antes si se administra el tratamiento.

\section{Bibliografía.}

Artamendi, S. F., R, J., Hermida, F., Secades-Villa, R., \& Garcia-Portilla, P. (Mayo de 2016). Cannabis y Salud Mental. Especial Psiquiatria, I(11).

Casas, C. R. (Agosto de 2017). Consumo de cannabis y psicopatología. Psicopatología, II(69).

Dimith, A. (Marzo de 2016). DSM-IV: Trastornos relacionados con sustancias. Trastornos relacionados con Cannabis. Estudio del Psioanálisis y psicología, VI(12).

E, Y., Casavielles, R., Herse, M. G., \& J, E. (junio de 2017). La marihuana y los efectos que provcan en los seres humanos. SciELO, XXI(3).

Gainza, I., Nogué, S., Velasco, C. M., Hoffman, R. S., Burillo-Putze, G., \& Dueñas., A. (Septiembre de 2015). Intoxicación por drogas. SciElo, XXVI(8).

Loirth, P. (Agosto de 2017). Trastornos relacionados con Sustancias (Adicciones). Revista de Salud Mental, II(13).

López-Ibor, J. J. (Julio de 2015). Trastornos relacionados con Cannabis. Clasificacion DSMIVTR, II(275).

Lorente, P., Actis, C., López-Cánovas, \& Hernández-Torrado. (Julio de 2016). Polaino Lorente, Chiclana Actis, López-Cánovas, Hernández-Torrado. ResearchGate, II(14).

Med, R. F. (Septiembre de 2018). El consumo del cannabis y sus repercusiones; información para el médico general. SciELO, $L X(5)$.

Organization, W. H. (07 de 2018). Efectos sociales y para la salud del consumo de cannabis sin fines médicos. Organización panamericana de la salud, II(76). 


\section{Trastornos causados por el consumo de sustancias derivadas del cannabis}

Vol. 3, núm. 3., (2019)

Mónica Monserrate Solórzano Vélez; Iván Andrés Jaramillo Chávez; Flor María Alcívar Chávez; Daniela Stefanía Cedeño Ching; Iris Galicia Cevallos Macías; María Belén Iglesias López

Patel, J., \& Marwaha., R. (Junio de 2019). Cannabis Use Disorder. StartPearls, II(16).

Pichot, P., Aliño, J. J.-I., \& Miyar, M. V. (Septiembre de 2017). Manual diagnóstico y estadistico de los trastornos mentales. Manual Diagnostico, III(317-319).

Royo, C., \& 1981, A. M. (Enero de 2014). Cannabis en los jóvenes: patología psiquiátrica y escalas de consumo. Tesis Doctorals en Xarxa, II(146).

T, S., WilkinsonRajiv, RadhakrishnanDeepak, \& D’Souza, C. (Junio de 2016). Impact of Cannabis Use on the Development of Psychotic Disorders. Spinger, I(17).

Tziraki, S. (Julio de 2017). Trastornos mentales y afectación neuropsicológica. Revista de Neurologia, $I I(11)$.

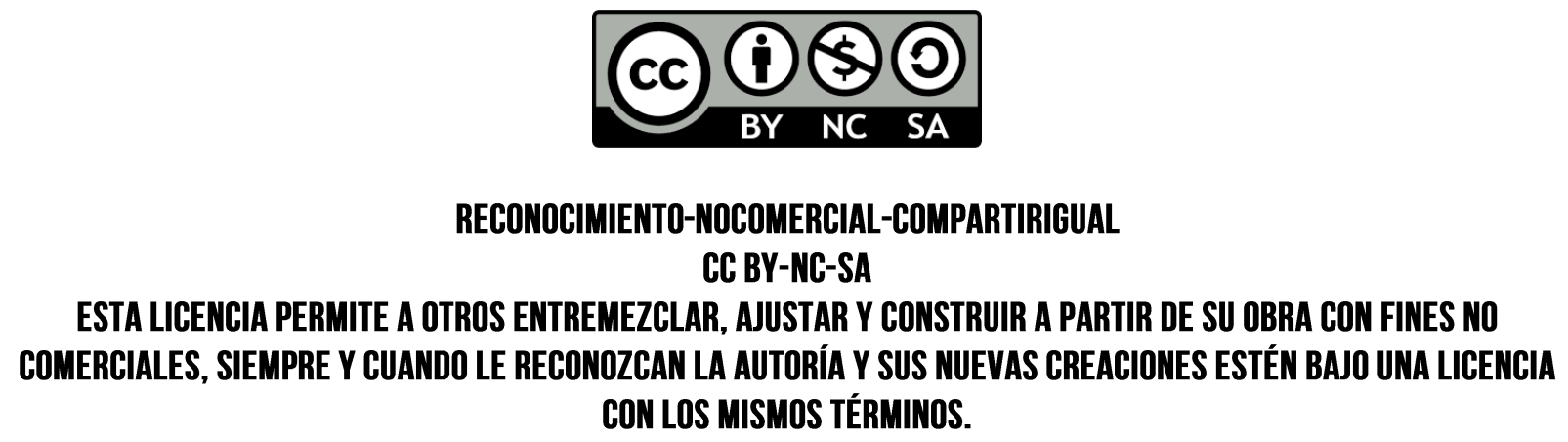

